\title{
Therapeutic Targeting of Circulating Tumor Cells: An Important Problem That Deserves Careful Study
}

In cancer research, the discovery and study of circulating tumor cells (CTCs) have seemed to open a world of possibilities. We now have the potential to gain cellular and molecular understanding of individual cases of metastatic cancer without invasive procedures. This area of research is, however, not without some basic pitfalls. In this commentary, we address some of these pitfalls by considering two recent examples in the published literature and discuss ways to overcome their limitations with the hope of informing those who may be entering the growing field of CTC research. Careful research design should always be followed to prevent incomplete or misleading studies from entering the literature, and thereby avoid setting back this burgeoning field.

Two recent CTC papers appearing in the journal PLOS ONE can serve as examples of preliminary studies lacking the necessary controls to yield meaningful results. In the first, Kim and Gaitas outline a creative application of photodynamic therapy (PDT) intended to prevent cancer metastasis, in which tubing could be attached to a patient's vasculature to divert blood into an external device where it would be exposed to LED illumination to induce cancer cell death. ${ }^{5}$ The illumination - combined with a photosensitizer conjugated to an antibody against CD44, a cell adhesion protein found on many cancer cells - is intended to eliminate CTCs in the blood. Their study attempts to examine the feasibility of this technique in vitro. Upon first glance the results of the paper might seem promising, with the vast majority of prostate cancer cells spiked into whole blood dying within an hour after $2 \mathrm{~min}$ treatment; however, the authors overlooked key aspects of treatment in blood that, when considered, significantly weaken the overall impact of the paper.

When critically evaluating this proposed therapy, one must first look at the feasibility of treating the entire blood volume in a realistic time frame. Limitations of throughput are a common issue in many proposed CTC technologies. Most devices test a small volume of blood and while the information gleaned from such volumes can be of scientific interest, ${ }^{4,10,13,14}$ the scale-up of these devices becomes more complicated. The scale-up of the PDT device to treat an adult blood volume $(\sim 5 \mathrm{~L})$ in this case does seem feasible, however. One may calculate that at a physiological blood velocity $(\sim 30 \mathrm{~cm} / \mathrm{s})$ typical of small arteries of equal diameter as the $1 \mathrm{~mm}$ internal diameter tubing used, 36 meters of tubing would be needed to achieve their prescribed 2 min of LED light exposure and thus the entire blood volume could be processed within about $6 \mathrm{~h}$. This amount of tubing would contain a blood volume of $28 \mathrm{~mL}$, also a reasonable design parameter. A single or double layer of this tubing could, in theory, be arranged in a small bedside or even portable extracorporeal device. Unfortunately no analysis of this form was presented in the 2015 paper. $^{5}$

Importantly, one must also consider the proposed therapy's effect on the normal blood cells, which would likewise also receive exposure to the photodynamic sensitizer and light source. This is the most problematic issue with the proposed technology. The authors chose CD44 as the molecular target for the photosensitizer therapy. While CD44 expression is well documented in cancer, ${ }^{3,6,7,11}$ it is also a major adhesion molecule for leukocytes. ${ }^{8,16} \mathrm{CD} 44$ goes by the alternate name phagocytic glycoprotein-1, and is a receptor for hyaluronic acid. The implication of this, of course, is that while the photosensitizer may target CTCs, leukocytes will almost certainly become bound to the sensitizer and also killed during light treatment. Furthermore, even if a more appropriate antigen was selected to better avoid off-target binding, the reactive oxygen species (ROS) generated by the therapy would still be likely to affect surrounding blood cells and accumulate within the plasma during the course of treatment. The authors argue that since the diffusion distance of ROS is small $(\sim 100 \mathrm{~nm}$, reportedly), the ROS generated at the surface of CTCs should not reach neighboring blood cells. While this might hold true under static conditions, under dynamic blood flow conditions it is the much greater convective length scale that must be considered when estimating whether ROS generated in the vicinity of one cell surface may affect neighboring cells. The chaotic mixing of bulk blood flow will nearly ensure that all cells within the peripheral blood flowing through the device will be equally exposed to ROS generated in the extracellular fluid. Remarkably, Kim and Gaitas demonstrated the ability to kill PC-3 cancer cells within spiked blood samples, but never tested whether the PDT approach 
was also killing blood cells at the same rate, a minimum requirement of any selective therapy designed to act in the bloodstream. ${ }^{5}$ In our opinion, this important control measurement should have been a necessary precursor to publication in a peer-reviewed research journal, and the absence of basic measurements such as these cast the overall conclusions into considerable doubt. The authors used the loss of fluorescence signal from PC-3 prostate cancer cells labeled with the live cell dye Calcein AM as their sole measure of cell death, whereas an additional assay of apoptosis and necrosis such as Annexin V, TUNEL, or MTT would be recommended, as well as the use of an additional cancer cell line.

Finally, whenever light is used to induce a cellular response, one must also be concerned with the effects of heating. This is particularly important in the blood since red blood cells will undergo lysis at temperatures above $45{ }^{\circ} \mathrm{C}$. ${ }^{2}$ Anywhere from 60 to $95 \%$ of the energy of LEDs is converted to heat. This heat is generally believed to not be given off as infrared heat and therefore may not affect the sample directly. However, there have been reports of IR heat production and resulting sample heating by LEDs, an effect that is dependent on the power used and the type of heat sink. It may thus be possible to avoid this issue through careful engineering, but heating should nevertheless have been considered and tested in the paper (it wasn't). All of the PDT experiments of Kim and Gaitas were performed in a foil-lined Styrofoam insulated cooler, using sealed, static well plates and blood-filled tubing sealed on both ends. Despite the diagram of their Figure 1, no flow experiments were performed. $^{5}$

The majority of current CTC research focuses on the isolation and enumeration of cells captured from patient blood samples for analysis. While this work does not directly involve the toxicity of reagents on either cancer or blood cells, it nevertheless holds its own pitfalls and issues that, if not properly addressed, can skew results and thereby lead to misleading conclusions.

One example of this is another paper by Gaitas and Kim published recently in PLOS ONE. ${ }^{1}$ The authors present a method for isolating CTCs in a flow device coated with antibody against epithelial cell adhesion molecule (EpCAM). The authors showed that they were able to capture $85 \%$ of EpCAM-expressing cancer cells suspended in buffer, and $44 \%$ of the cancer cells from whole blood spiked with cancer cells. This straightforward method shares similarities with other EpCAM-antibody isolation approaches previously described in the literature, ${ }^{4,12,14,15}$ and would likely not encounter major challenges in capturing those CTCs that highly express EpCAM. However, as with the previously discussed paper by Kim and Gaitas, some much needed control experiments and other considerations were neglected, seriously compromising the soundness of the study.

First, the device is presented as a dynamic microfluidic device intended to capture cells from the bloodstream of patients to achieve a therapeutic filtering of the blood, however the only experiments performed in the study were under static (i.e., no flow) conditions. It is likely that the addition of shear force would affect the capture efficiency of the device and this is something that should have been addressed to support the conclusions of the paper.

Second, the authors again failed to take the blood cells into consideration. In other CTC isolation studies, the capture purity as well as the capture efficiency is reported. ${ }^{4,9,13}$ This is important for two reasons. First, if too many white blood cells adhere to the device, they will block the capture of further cancer cells, thereby decreasing the capture efficiency. This is especially important for the device discussed in the Gaitas and Kim paper, as they are suggesting that this technology could be used as a blood-filtering device for metastatic cancer patients. Such a device would process many more white blood cells than cancer cells $\left(>10^{5}\right.$-fold), and over time the device would likely become less and less efficient due to nonspecific adhesion of leukocytes. The purity is equally important for post-isolation processing. Certain techniques that involve DNA and RNA analysis require relatively pure samples to detect cancer-associated mutations without excessive contamination from blood cell genetic material. For these reasons, the achieved purity is always reported for new CTC isolation devices.

We were quite surprised that Gaitas and Kim only reported the number of cancer cells captured by their antibody-coated tubing and did not attempt to assess the number of leukocytes captured, either qualitatively with a bright field or phase contrast micrograph to accompany the fluorescence image showing EpCAM + capture, or quantitatively using a hemocytometer or flow cytometer. We are even more surprised that such a preliminary effort would be accepted for publication in a reputable journal such as PLOS ONE. Surely a study whose abstract's first sentence promises a "tube to selectively capture circulating tumor cells" should show some measure of selectively within its pages? Perhaps bright field images showed a degree of indiscriminate cell capture which contradicted the stated goals of the study. The authors' sly addition of the phrase "In this preliminary effort" to the second sentence of both abstracts should not justify the publication of such incomplete work.

As the title of this commentary states, the development of new technology to study (and treat) circulating 
tumor cells is sorely needed, but it must be carried out with sufficient rigor to advance the field. Authors, reviewers, and editors should work together to avoid populating the literature with hastily done, inadequate work that acts more to mislead and confuse rather than enlighten. We also hope that our commentary can serve a more productive purpose than simply singling out one laboratory that is new to the CTC field, by pointing out some of the common pitfalls and necessary considerations for researchers to be aware of. Overall, we are encouraged that the field of CTC research is growing and more innovative ideas are being proposed, such as blood-filtering devices and new CTC-targeted therapies. These ideas are vital for the advancement of cancer metastasis research and treatment. By addressing common issues such as those presented in this commentary, we believe that CTC research can become a more important area of cancer investigation. We hope those who are just entering the field can draw inspiration and advice from this commentary and we look forward to seeing what technologies the next wave of CTC research will bring.

\section{REFERENCES}

${ }^{1}$ Gaitas, A., and G. Kim. Chemically modified plastic tube for high volume removal and collection of circulating tumor cells. PLoS One 10:e0133194, 2015.

${ }^{2}$ Gershfeld, N. L., and M. Murayama. Thermal instability of red blood cell membrane bilayers: temperature dependence of hemolysis. J. Membr. Biol. 101:67-72, 1988.

${ }^{3}$ Hanley, W. D., M. M. Burdick, K. Konstantopoulos, and R. Sackstein. CD44 on LS174T colon carcinoma cells possesses E-selectin ligand activity. Cancer Res. 65:58125817, 2005.

${ }^{4}$ Hughes, A. D., J. Mattison, L. T. Western, J. D. Powderly, B. T. Greene, and M. R. King. Microtube device for selectin-mediated capture of viable circulating tumor cells from blood. Clin. Chem. 58:846-853, 2012.

${ }^{5} \mathrm{Kim}$, G., and A. Gaitas. Extracorporeal photo-immunotherapy for circulating tumor cells. PLoS One 10:e127219, 2015.

${ }^{6}$ Li, X.-P., X.-W. Zhang, L.-Z. Zheng, and W.-J. Guo. Expression of CD44 in pancreatic cancer and its significance. Int. J. Clin. Exp. Pathol. 8:6724-6731, 2015.
${ }^{7}$ Maeda, S., et al. CD44 and CD133 expressions in primary tumor cells correlate to survival of pancreatic cancer patients. Open Surg. Oncol. J. 1:1-7, 2009.

${ }^{8}$ McDonald, B., and P. Kubes. Interactions between CD44 and hyaluronan in leukocyte trafficking. Front. Immunol. 6:68, 2015.

${ }^{9}$ Nagrath, S., et al. Isolation of rare circulating tumour cells in cancer patients by microchip technology. Nature 450:1235-1239, 2007.

${ }^{10}$ Okegawa, T., K. Hayashi, H. Hara, K. Nutahara, and E. Higashihara. Immunomagnetic quantification of circulating tumor cells in patients with urothelial cancer. Int. J. Urol. 17:254-258, 2010.

${ }^{11}$ Ouhtit, A., Z. Y. Abd Elmageed, M. E. Abdraboh, T. F. Lioe, and M. H. G. Raj. In vivo evidence for the role of CD44s in promoting breast cancer metastasis to the liver. Am. J. Pathol. 171:2033-2039, 2007.

${ }^{12}$ Sieuwerts, A. M., et al. Anti-epithelial cell adhesion molecule antibodies and the detection of circulating normal-like breast tumor cells. J. Natl. Cancer Inst. 101:61-66, 2009.

${ }^{13}$ Stott, S. L., et al. Isolation of circulating tumor cells using a microvortex-generating herringbone-chip. Proc. Natl. Acad. Sci. U. S. A. 107:18392-18397, 2010.

${ }^{14}$ Stott, S. L., et al. Isolation and characterization of circulating tumor cells from patients with localized and metastatic prostate cancer. Sci. Transl. Med. 2:25ra23, 2010.

${ }^{15}$ Thege, F. I., et al. Microfluidic immunocapture of circulating pancreatic cells using parallel EpCAM and MUC1 capture: characterization, optimization and downstream analysis. Lab Chip 14:1775-1784, 2014.

${ }^{16}$ Zuchtriegel, G., et al. Spatiotemporal expression dynamics of selectins govern the sequential extravasation of neutrophils and monocytes in the acute inflammatory response. Arterioscler. Thromb. Vasc. Biol. 35:899-910, 2015.

JOCELYN R. MARSHALL

Meinig School of Biomedical Engineering Cornell University, Ithaca, NY, USA

\section{Michael R. King}

Meinig School of Biomedical Engineering Cornell University

Electronic mail: mrk93@cornell.edu 